\title{
The 2006 Data Expo of the American Statistical Association
}

\author{
Paul Murrell
}

Received: 15 June 2010 / Accepted: 2 July 2010 / Published online: 11 July 2010

(C) Springer-Verlag 2010

\section{Introduction}

Since 1983, the Sections on Statistical Computing and Graphics of the American Statistical Association have held a Data Exposition competition as part of the Joint Statistical Meetings (JSM). These competitions presented entrants with a data set and challenged them to produce a comprehensive analysis of the data in poster form, with an emphasis on graphical exploration and presentation of results. Early Data Expos were held roughly every 2 years (a full list can be found at http://stat-computing.org/ dataexpo/), but there was a hiatus after 1997.

At the 2006 Joint Statistical Meetings (JSM) conference in Seattle, the Data Exposition competition was revived, with help from the Section on Statistics and the Environment, using a data set of atmospheric measurements from NASA. Fourteen groups submitted entries and the following articles provide a platform for the authors of the top three entries in the Data Expo competition to describe their winning analyses in more detail.

\section{Overview of the data}

The data for the Data Expo were geographic and atmospheric measures on a regular, but very coarse 24 by 24 grid, with a resolution of 2.5 degrees of latitude/longitude, covering Central America (see Fig. 1). The variables were: elevation, temperature (surface and air), ozone, air pressure, and cloud cover (low, mid, and high). The temperature values are in Kelvin, where degrees Celsius $=$ degrees Kelvin -273.15 . Cloud

P. Murrell $(\bowtie)$

Department of Statistics, The University of Auckland, Private Bag 92019,

Auckland, New Zealand

e-mail: paul@stat.auckland.ac.nz 


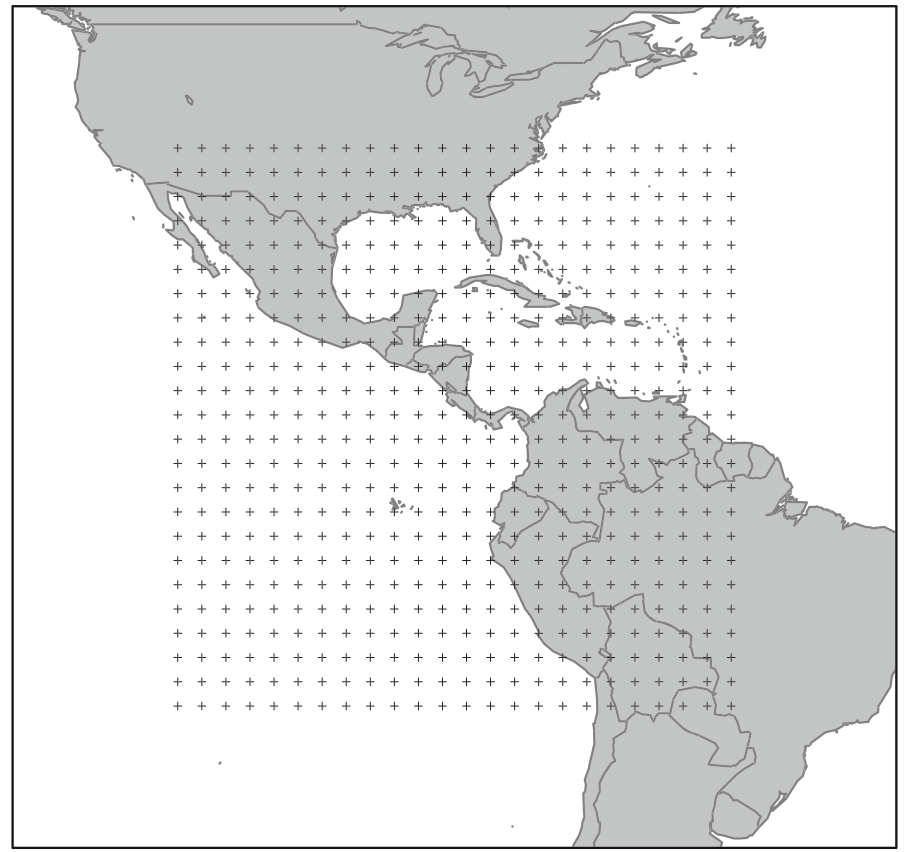

Fig. 1 This map shows the area over which the atmospheric data were collected for the Data Expo dataset. The data values represent averages over large geographical regions covering 2.5 degrees of latitude/longitude. The crosses on the map indicate the centres of these regions

cover is measured as the monthly mean percent of the sky covered by clouds at different altitudes; low-cloud is below $3.24 \mathrm{~km}$, mid-cloud is between 3.24 and $6.5 \mathrm{~km}$, and high-cloud is above $6.5 \mathrm{~km}$. Elevations are in meters, pressure values are in millibars and ozone abundance is measured in "Dobson" units.

With the exception of elevation, all variables were monthly averages, with observations for January 1995 to December 2000. These data were obtained from the NASA Langley Research Center Atmospheric Sciences Data Center's Live Access Server http://mynasadata.larc.nasa.gov/las/servlets/dataset.

The data were provided as text files, with one file for each variable for each month of data, plus elevation data at two different resolutions (a total of 506 files). The files obtained from NASA contained highly processed and aggregated values based on original satellite/tracking station data, in some cases combining multiple sources, and in some cases interpolating from historical and survey data. There was no guarantee that the data values for even a single variable were from a single, consistent source.

\section{The data expo challenge}

The Data Expo challenge was a very general one: to produce a summary of the important features of the data, with an emphasis on graphical displays. The multivariate, 
spatial, and temporal aspects of the data provided plenty of challenges and all of the winning entries demonstrated sophisticated and novel approaches, both in terms of graphical and computational techniques.

For more details about the competition, including poster materials from all of the entries, see the Data Expo web site http://stat-computing.org/dataexpo/2006/entries. html.

\section{The winning entries}

The following three articles present the results from the first, second, and third placed entries in the JSM 2006 Data Expo. The winning entry was from Sang-Hoon Cho and Hyonho Chun (Cho and Chun 2010), both of whom were graduate students at the University of Wisconsin. Second place went to Jonathon Hobbs, Hadley Wickham, Heike Hoffman, and Di Cook (Hobbs et al. 2010) from Iowa State University (the first two authors were again graduate students at the time of the Data Expo). The third place entry was by a team from Vanderbilt University: Svetlana Eden, Theresa Scott, Angel An, Jeffrey Horner, and Cathy Jenkins (Eden et al. 2010).

These winners were chosen because they stood out both in terms of the graphical, computational, or methodological approach that was taken and in terms of the visual impact of the poster itself. The first prize was awarded to Cho and Chun (2010) because, of the three top posters, they had been most successful at communicating the results of their analyses.

The judges' decisions were based on the posters that were presented at JSM 2006, but each of the articles that follow have, to varying extents, updated and added to the analyses that were presented on the posters.

The articles vary enormously in the approach taken to the problem, no doubt partly as a result of the very general problem statement and the high dimensionality and complexity of the data set. However, this variety also nicely reflects the fact that every individual analyst will always bring a certain perspective to a data analysis problem, something that the team from Iowa State acknowledge occurred even within their own team.

The submission by Eden et al. (2010) places a stronger focus on the tools and on the process used to visualize the data set, with less emphasis on the features of the data set itself. They provide useful demonstrations of plots based on polar coordinates and plots made up of small-multiples Tufte (1990).

The submission from Hobbs et al. (2010) also emphasizes the process involved in their data analysis, but in addition they present a number of interesting features that they discovered in the data set. This article is also notable for its use of interactive plots as well as static plots. It is an impressive demonstration of multivariate data analysis.

Cho and Chun (2010) focus much more on the specific techniques that were applied to the specific goals of their analysis. They also deal only with subsets of the data, but the analysis involves more sophisticated techniques than were employed in the other submissions. The result is an interesting view of some of the finer details of the data set. 


\section{References}

Cho SH, Chun H (2010) Visualizing abnormal climate change in central America from 1995 to 2000. Computational Statistics Insert Doi

Eden SK, An AQ, Horner J, Jenkins C, Scott TA (2010) A two-step process forgraphically summarizing spatial temporal multivariate data in two dimensions. Computational Statistics Insert Doi

Hobbs J, Wickham H, Hoffman H, Cook D (2010) Glaciers melt as mountains warm: A graphical case study. Computational Statistics Insert Doi

Tufte ER (1990) Envisioning information. Graphics Press, Cheshire 